\title{
A Self-Limited Facial Rash in a Lupus Patient: The Case of Primary Facial Raynaud's Phenomenon
}

\author{
Kamel El-Reshaid ${ }^{a}$ Shaikha Al-Bader ${ }^{b}$ Hossameldin Tawfik Sallam ${ }^{b}$ \\ ${ }^{a}$ Department of Medicine, Faculty of Medicine, Kuwait University, Safat, Kuwait; \\ bepartment of Medicine, Nephrology Unit, Amiri Hospital, Ministry of Health, Kuwait City, \\ Kuwait
}

\section{Keywords}

Autoimmunity $\cdot$ Face $\cdot$ Raynaud's $\cdot$ Skin $\cdot$ Systemic lupus erythematosis

\begin{abstract}
Skin is involved in $80 \%$ of systemic lupus erythematosus (SLE) and the second most affected after joint disease. Lupus-specific lesions include (a) acute ones viz. malar rash (80\%), (b) subacute ones viz. photosensitive maculopapular dermatitis (50\%), and (c) chronic ones viz. discoid rash. The lupus nonspecific lesions include; (a) nonscarring alopecia (86.67\%), oral ulcers (56.67\%), vasculitic lesions (33.34\%), bullous lesions (10\%), and Raynaud's phenomenon (6.67\%). In this case report, we describe a patient with SLE and antiphospholipid antibodies that had developed a transient facial form of Raynaud's phenomenon that was not associated with disease activity and digital changes. Its association with SLE is discussed.
\end{abstract}

\section{Introduction}

Systemic lupus erythematsus (SLE) is a chronic autoimmune disease characterized by autoantibody production, inflammation, and tissue damage in multiple organs resulting from the activation of numerous pro-inflammatory pathways [1]. The etiology of SLE is not fully understood, but both genetic predisposition and environmental triggers are believed to be involved [2]. Women are more frequently affected than men for every age and ethnic group [3]. The disease is more common in Northern America and especially in blacks and Asian ethnicity with an incidence at 23.2/100,000 person/years and prevalence at 241/100,000 

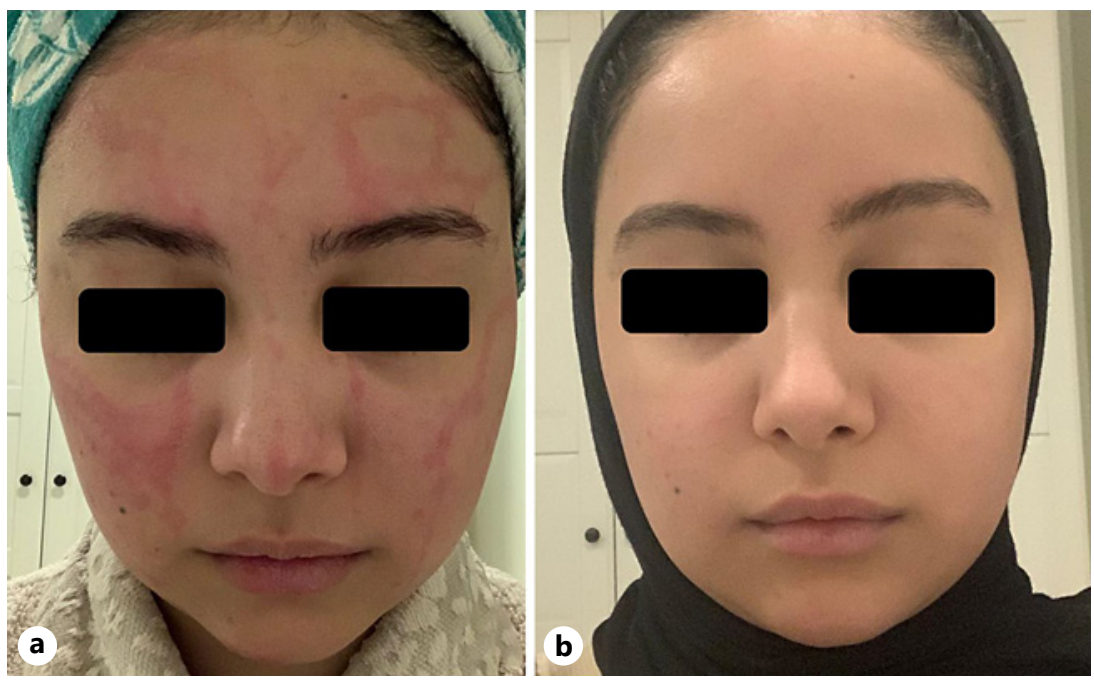

Fig. 1. Front-view of the patient with the initial self-limited Raynaud's rash (a) and hours later (b).

people [4]. Clinical manifestations vary widely among patients and commonly include rash, arthritis, and nephritis, but the central nervous system and other major organ systems can also be affected [5]. Skin and mucous membrane are involved in over $80 \%$ of patients with SLE and hence are the second most commonly affected organ after joint involvement [6]. Lupus-specific lesions include (a) acute ones viz. malar rash (80\%), (b) subacute ones viz. photosensitive maculopapular dermatitis (50\%), and (c) chronic ones viz. discoid rash. The lupus nonspecific lesions include (a) nonscarring alopecia (86.67\%), oral ulcers (56.67\%), vasculitic lesions (33.34\%), bullous lesions (10\%), and Raynaud's phenomenon (6.67\%) [7]. Cutaneous lesions are important as a diagnostic aid as these account for 4 out of 11 revised ARA criteria for disease classification. Moreover, lupus-specific skin lesions indicate severe systemic involvement, whereas nonspecific ones are associated with disease flare [8]. In this case report, we describe a patient with SLE and antiphospholipid antibodies that had developed a transient facial form of Raynaud's phenomenon that was not associated with disease activity and digital changes.

\section{The Case}

A 28-year-old woman presented with nonpruritic yet painful reddish rash over her face immediately after having a warm shower in autumn. The rash persisted for few hours then disappeared completely (Fig. 1-3). Past history was significant for systemic lupus erythematosis that started 13 years ago. She had fever, arthritis, maculopapular facial skin rash, and pancytopenia, renal failure with hypertension, fluid overload, azotemia, and proteinuria. Moreover, she had pericardiomyocarditis and neuropsychiatric abnormalities with abnormal EEG spikes in the frontotemporal lobes. Diagnosis of SLE was established with high ANA and anti-dsDNA titers and low serum complements 3 and 4. Moreover, she had high IgG anticardiolipin antibodies. Her kidney biopsy was consistent with grade 4 lupus nephritis. ANCA, anti Scl-70 (topoisomerase I), anti-Ro (SSA) and anti-La (SSB), anti-GBM antibodies, hepatitis B surface antigen, and anti-hepatitis C antibodies were negative. She was treated with 3 consecutive days of $1 \mathrm{~g}$ of Solumedrol followed by Prednisone $60 \mathrm{mg}$ daily in addition to Mycophenolate mofetil $1 \mathrm{~g}$ twice daily. She improved 

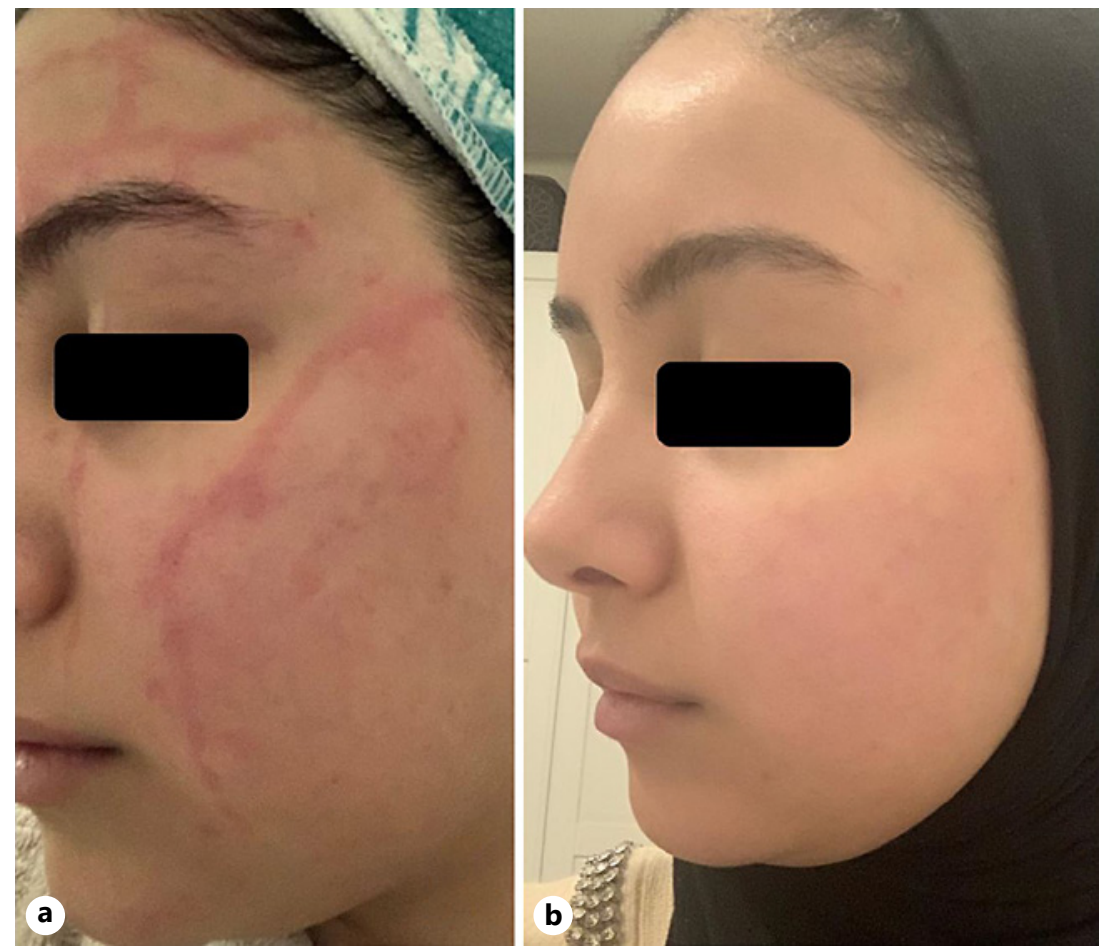

Fig. 2. Left side view of the patient with the initial self-limited Raynaud's rash (a) and hours later (b).

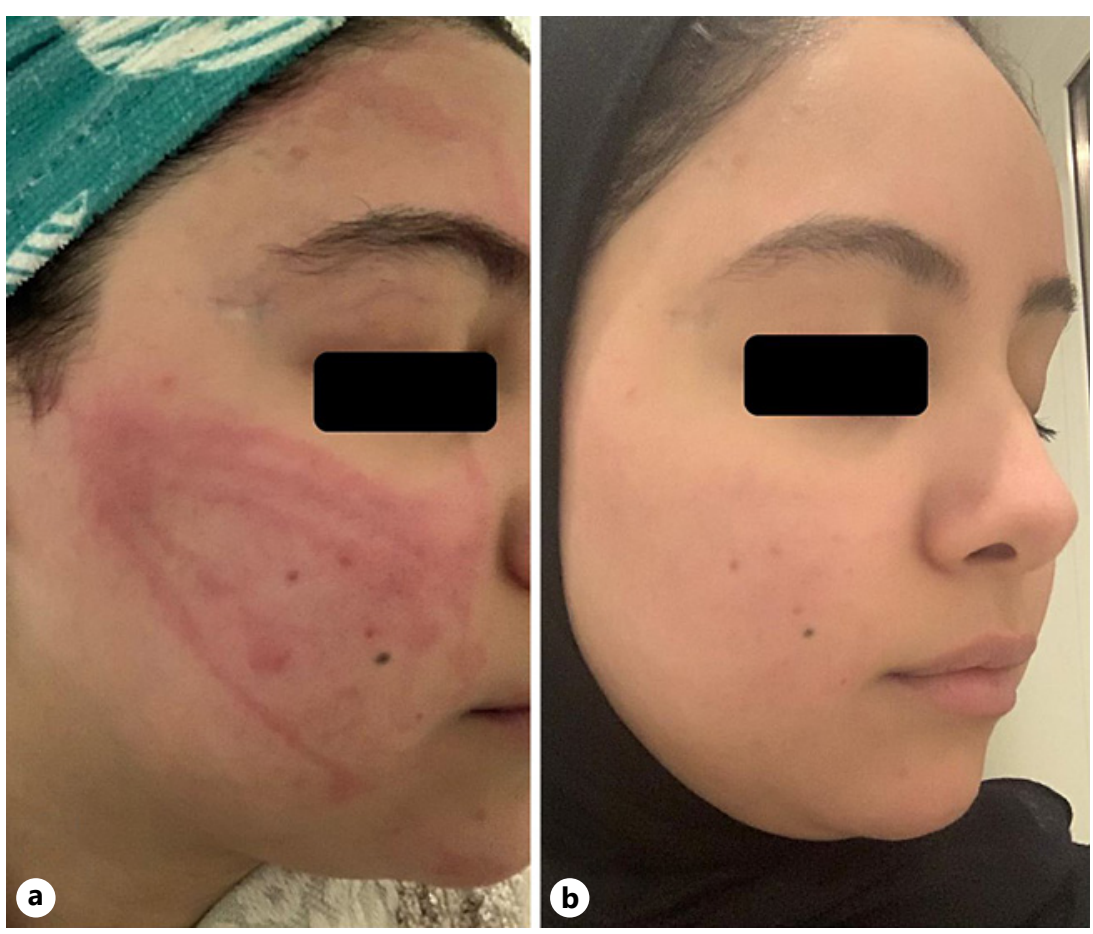

Fig. 3. Right side view of the patient with the initial self-limited Raynaud's rash (a) and hours later (b). 
over the past 3 months and was maintenance dose of Mycophenolate $1 \mathrm{~g}$ twice daily with P $5 \mathrm{mg}$ daily since then. For her neuropsychiatric manifestations and positive antiphospholipids, she was kept on Kepra 500 mg twice daily and Warfarin 5 mg daily for 2 years. On evaluation, she was asymptomatic and did not have any changes in her hands. Vital signs, general examination, and systemic ones did not show abnormality. Laboratory investigations were within normal ranges with regards complete blood counts as well as renal, liver, and lipid profile. Urine routine and microscopy were normal. ANA, antidsDNA, and serum complements were normal. Capillaroscopy did not show abnormalities in nail-bed vessels. Subsequently, and up to 6 months, she did not have similar attacks or hand changes.

\section{Discussion}

Raynaud's phenomenon is a cutaneous vascular disorder which presents with intermittent triphasic changes in the color of the extremities (fingers, toes, nose, cheeks, and ears), usually triggered by cold exposure or emotional stress. They change from white (owing to vasoconstriction) to blue (tissue hypoxia) and red on rewarming (reperfusion). This may last for minutes to hours and can be painful, particularly in the rewarming phase [9]. Raynaud's is a common disorder. About 1 in 20 people develop Raynaud's phenomenon. In $80-90 \%$ of patients with Raynaud's phenomenon, there is no underlying cause established [10]. Secondary causes include (a) compression by a cervical rib in unilateral cases, (b) SLE and scleroderma, (c) systemic vasculitis and leukocytoclastic and urticarial ones, (d) thrombotic vasculopathy, (e) erythromelalgia, (f) hands over-use or vibration tools, and (g) medications viz. beta blockers, ergotamine, sumatriptan, and chemotherapeutics [11]. Primary Raynaud's usually first develops in teenagers and young adults, but it can develop at any age. Secondary Raynaud's can develop at any age when the underlying condition develops. Features that may suggest secondary Raynaud's include (a) onset of symptoms after 30 years of age, (b) abrupt onset with rapid progression and worsening of symptoms, (c) severe symptoms that may include an ulcer or gangrene of part of a finger or toe, (d) abnormal nail-bed vessels on capillaroscopy [12]. The latter is an indicator of chronic sclerotic vasculopathy rather than shortlived arterial spasm in the primary form [11]. In SLE, such sclerotic changes have been attributed to IgG skin deposits where macrophages and signaling of TNF $\alpha$ /TNFR1 and IFN/ IFNR play an important role. The intracellular molecules including Syk and calcium/ calmodulin 4 and NFAT are involved in the manifestation of skin damage in lupus-prone mice. $\mathrm{UV}$ is the most typical environmental factor to trigger skin injury in areas of IgG deposition in SLE [13]. Interestingly, the proposed mechanism of RF is mediated by autoimmune deregulation involving cytokeratin 10 (K10) as a candidate autoantigen; their study in mice showed that anti-K10 antibodies can mediate ischemia similar to that seen in Raynaud phenomenon [14]. Hence, its association with serious autoimmune disease should not be considered a cause and an effect or an indication of its activity. Our patient had a single self-limited attack of RF without disease activity and without capillaroscopic defects. The presentation favors primary RF rather than secondary to her SLE disease. In conclusion, our case report is unique with (a) primary form of RF in a patient with autoimmune disease and (b) facial manifestation without digits disease.

\section{Acknowledgement}

The authors have no acknowledgment to be stated.

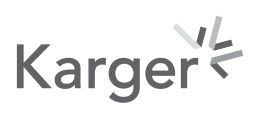


El-Reshaid et al.: Raynaud's and SLE

\section{Statement of Ethics}

There was no harmful investigation, unethical new treatment conducted on this patient that needed approval of an Ethical Committee. It was a simple report on a rare phenomenon in a patient. Moreover, the patient accepted, in written, publication of the details of her medical case and agreed on providing censored pictures of her phenomenon.

\section{Conflicts of Interest Statement}

The authors have no conflicts of interest to declare.

\section{Funding Sources}

The authors have no funding sources to declare.

\section{Author Contributions}

Dr. Shaikha al-Bader is the nephrologist responsible for the patient and all her previous and current treatment. She was the one who consulted Prof. Kamel El-Reshaid for the new phenomenon.

Prof. Kamel El-Reshaid identified the phenomenon as a unique case of isolated facial Raynaud's without digital involvement. Moreover, he identified the case as a primary and not secondary to her SLE (see text). He planned the investigations and guided the team. He wrote the draft of the article and added the inputs of the 2 co-authors. He is also the corresponding author on this article to the journal. Dr. Hossameldin conducted extensive search on the subject and did capillaroscopy for the patient.

All the authors satisfied the ICMJE criteria for author's contributions as they:

1. had substantial contributions to the conception, analysis, and interpretation of data.

2. Dr. Shaikha provided data on the stability of the patient and to confirm that the case is primary not due to active SLE disease. Dr. Hossameldin provided research data and confirmed the absence of digital disease by capillaroscopy.

3. All the 3 authors approve the final version to be published.

4. All the 3 authors agreed to be accountable for all aspects of the work in ensuring that questions related to the accuracy or integrity of any part of the work are appropriately investigated and resolved.

\section{Availability of Data and Material}

The data and images of this study are openly available with Prof. Kamel El-Reshaid (corresponding author) and further enquiries can be directed to him.

\section{References}

1 Liu Z, Davidson A. Taming lupus: a new understanding of pathogenesis is leading to clinical advances. Nat Med. 2012;18(6):871-82.

2 Cooper GS, Dooley MA, Treadwell EL, St Clair EW, Parks CG, Gilkeson GS. Hormonal, environmental, and infectious risk factors for developing systemic lupus erythematosus. Arthritis Rheum. 1998;41(10):1714-24. 
3 Strickland FM, Hewagama A, Lu Q, Wu A, Hinderer R, Webb R, et al. Environmental exposure, estrogen and two $\mathrm{X}$ chromosomes are required for disease development in an epigenetic model of lupus. J Autoimmun. 2012;38(2-3):J135-43.

4 Rees F, Doherty M, Grainge MJ, Lanyon P, Zhang W. The worldwide incidence and prevalence of systemic lupus erythematosus: a systematic review of epidemiological studies. Rheumatol. 2017;56(11):1945-61.

5 Rahman A, Isenberg DA. Systemic lupus erythematosus. N Engl J Med. 2008;358(9):929-39.

6 McCauliffe DP. Cutaneous lupus erythematosus. Semin Cutan Med Surg. 2001;20(1):14-26.

7 Alakes K, Alakendue G. Cutaneous manifestations of systemic lupus erythematosis in a tertiary referral center. Indian J Dermatol. 2009;54:132-136.

8 Zecević RD, Vojvodić D, Ristić B, Pavlović MD, Stefanović D, Karadaglić D. Skin lesions: an indicator of disease activity in systemic lupus erythematosus? Lupus. 2001;10(5):364-7.

9 Wigley FM, Flavahan NA. Raynaud's phenomenon. N Engl J Med. 2016;375(6):556-65.

10 Suter LG, Murabito JM, Felson DT, Fraenkel L. The incidence and natural history of Raynaud's phenomenon in the community. Arthritis Rheum. 2005;52(4):1259-63.

11 Pauling JD, Hughes M, Pope JE. Raynaud's phenomenon: an update on diagnosis, classification and management. Clin Rheumatol. 2019;38(12):3317-30.

12 Smith V, Herrick AL, Ingegnoli F, Damjanov N, De Angelis R, Denton CP, et al. EULAR Study Group on Microcirculation in Rheumatic Diseases and the Scleroderma Clinical Trials Consortium Group on Capillaroscopy. Standardisation of nailfold capillaroscopy for the assessment of patients with Raynaud's phenomenon and systemic sclerosis. Autoimmun Rev. 2020;19:102458.

13 Deng GM. Pathogenesis of Skin Injury of Systemic Lupus Erythematosus. Curr Rheumatol Rep. 2018;20(2):5.

14 Ascherman DP, Zang Y, Fernandez I, Clark ES, Khan WN, Martinez L, et al. An Autoimmune Basis for Raynaud's Phenomenon: Murine Model and Human Disease. Arthritis Rheumatol. 2018;70(9):1489-99. 